\title{
FIBER HOMOTOPIC TRIVIAL BUNDLES OVER COMPLEX PROJECTIVE SPACES
}

\author{
KEE YUFN IAM ${ }^{1}$
}

\begin{abstract}
We give a more illuminating derivation of a wellknown condition (obtained by $M$. Atiyah and J. A. Todd) for certain vector bundles over complex projective spaces to be stably fiber homotopic trivial, together with a generalization.
\end{abstract}

Let $H$ be the Hopf line bundle over the complex projective space $C P^{k-1}$ and $n H$ the $n$-fold Whitney sum of $H$ with itself. In [2], it is shown that a necessary condition for $n H$ to be stably fiber homotopic trivial is that $n$ be divisible by the "Atiyah-Todd number" $M_{k}$, defined as follows: for a prime $p$, the highest power of $p$ dividing $M_{k}$ is

$$
v_{p}\left(M_{k}\right)=\operatorname{Max}\left\{r+v_{p}(r): 1 \leqq r \leqq[(k-1) /(p-1)]\right\} .
$$

In [1], this condition is shown to be sufficient as well.

The number-theoretic derivation of $M_{k}$ in [2] is somewhat involved. We present a simpler, and more illuminating, derivation.

Recall $K\left(C P^{k-1}\right)=Z[x] / x^{k}$ where $x=H^{*}-1$. For $n H$ to be stably fiber homotopic trivial, the "cannibalistic classes" $\theta_{p}(n H)$ must satisfy (see $[4$, p. 37])

$$
\theta_{p}(n H)=p^{n} u / \psi^{\nu}(u),
$$

where $u$ is some element in $1+\tilde{K}\left(C P^{k-1}\right)$. But by [4, p. 34],

$$
\begin{aligned}
\theta_{p}(n H) & =\left(\theta_{x}(H)\right)^{n}=\left[\left((x+1)^{p}-1\right) / x\right]^{n} \\
& =\left(\left(\begin{array}{l}
p \\
1
\end{array}\right)+\left(\begin{array}{l}
p \\
2
\end{array}\right) x+\cdots+\left(\begin{array}{c}
p \\
p-1
\end{array}\right) x^{p-2}+x^{p-1}\right)^{n} \\
& =\left(p y+x^{p-1}\right)^{n},
\end{aligned}
$$

where $y$ is an element in $1+\tilde{K}\left(C P^{k-1}\right)$. Since the elements $y^{n-r} x^{(p-1) r}$ $(1 \leqq r \leqq[(k-1) /(p-1)])$ form a partial basis of $\widetilde{K}\left(C P^{k-1}\right)$, we conclude

Received by the editors October 27, 1971.

AMS 1970 subject classifications. Primary 55F50; Secondary 55F40.

Key nords and phrases. Complex projective spaces, stably fiber homotopic trivial bundles, "Atiyah-Todd" number, cannibalistic classes.

${ }^{1}$ Supported by the National Research Council of Canada (Grant No. 67-7562).

(c) Americar Mathematical Society 1972 
that

$$
p^{r} \mid\left(\begin{array}{l}
n \\
r
\end{array}\right) \text { for all } 1 \leqq r \leqq\left[\frac{k-1}{p-1}\right] .
$$

The binomial identity

$$
\left(\begin{array}{c}
m+n \\
r
\end{array}\right)=\sum_{i+j=r}\left(\begin{array}{c}
m \\
i
\end{array}\right)\left(\begin{array}{l}
n \\
j
\end{array}\right)
$$

shows that, for $p$ a fixed prime, all the $n$ 's satisfying $(*)$ form an ideal. Direct inspection from the formula

$$
\left(\begin{array}{l}
n \\
r
\end{array}\right)=\frac{n}{r}\left(\begin{array}{l}
n-1 \\
r-1
\end{array}\right)=\frac{n}{r} \prod_{j=1}^{r-1}\left(\frac{n-j}{j}\right)
$$

shows that $p^{\lambda}$ belongs to this ideal iff $\lambda \geqq v_{p}\left(M_{k}\right)$. This completes the derivation of $M_{k}$.

Notice that if ordinary characteristic classes (or equivalently, Steenrod reduced $p$ th power operations on Thom classes) were used instead of $\theta_{p}$, one would obtain (cf. [3, p. 445]) the following weaker necessary condition:

$$
p \mid\left(\begin{array}{l}
n \\
r
\end{array}\right) \text { for all } 1 \leqq r \leqq\left[\frac{k-1}{p-1}\right] .
$$

Thus our derivation brings out the superiority of $K$-theory methods.

Let $h=q^{\alpha}$ be a prime power. By the same kind of argument, it can be shown that a necessary condition for $n H^{h}$ to be stably fiber homotopic trivial is

$$
\begin{aligned}
& v_{p}(n) \geqq v_{p}\left(M_{k}\right) \quad \text { for any prime } p \neq q ; \text { and } \\
& v_{p}(n) \geqq \operatorname{Max}\left\{r+v_{p}(r): 1 \leqq r \leqq[(k-1) / h(p-1)]\right\} \quad \text { for } p=q .
\end{aligned}
$$

The method of [1] can then be used to establish that this is a sufficient condition as well. Thus the order of $J\left(H^{h}\right)$ in the group $J\left(C P^{k-1}\right)$ is completely determined.

\section{REFERENCES}

1. J. F. Adams and G. Walker, On complex Stiefel manifolds, Proc. Cambridge Philos. Soc. 61 (1965), 81-103. MR 30 \#1516.

2. M. F. Atiyah and J. A. Todd, On complex Stiefel manifolds, Proc. Cambridge Philos. Soc. 56 (1960), 342-353. MR 24 \#A2392.

3. A. Borel and J.-P. Serre, Groupes de Lie et puissances réduites de Steenrod, Amer. J. Math. 75 (1953), 409-448. MR 15, 338.

4. R. Bott, Lectures on $K(X)$, Math. Lecture Note Series, Benjamin, New York, 1969. MR 41 \#2667.

Department of Mathematics, University of British Collmbia, Vancouver 8 , B.C., Canada 